\title{
"Quadruple hamstring tendon graft versus Bone-Patellar- Tendon-Graft for arthroscopic Anterior Cruciate Ligament reconstruction- comparison study with follow up of 2 years."
}

\author{
Dr. Nikhil Joseph Martin ${ }^{1 *}$, Dr. Shishir.S.M², Dr. Kanagasabai.R ${ }^{3}$, \\ Dr. Syed Najimudeen ${ }^{4}$, Dr. James J Gnanadoss ${ }^{5}$. \\ ${ }^{1 *}$ Junior Consultant, M.S Ortho, Department of Orthopaedics, Medicare Hospital, Kodungallur-680664,India. \\ ${ }^{2-}$ Assistant Professor, M.S.Ortho, D.N.B.,M.Ch.(ortho),Department of Orthopaedics, Pondicherry Institute of \\ Medical Sciences, Kalapet,Pondicherry-605014,India. \\ ${ }^{3-}$ Professor, M.S.Ortho, Department of Orthopaedics, Pondicherry Institute of Medical Sciences, Kalapet, \\ Pondicherry-605014, India. \\ ${ }^{4-}$ HOD and Professor, M.S.Ortho,Department of Orthopaedics, Pondicherry Institute of Medical Sciences, \\ Kalapet,Pondicherry 605014,India. \\ ${ }^{5-}$ Professor, M.S.Ortho ,Department of Orthopaedics, Pondicherry Institute of Medical Sciences, Kalapet, \\ Pondicherry-605014,India.
}

\begin{abstract}
:
Introduction: Anterior Cruciate Ligament (ACL) tear is the most common ligamentous injury to the knee joint. Two most commonly used grafts for reconstruction are Quadruple Hamstring tendons (QHT) and Bone Patellar Tendon Bone (BPTB) graft. Graft fixation techniques vary with different surgeons. Aim of this study was to compare the clinical outcome of reconstruction of ACL using QHT and BPTB grafts performed by single surgeon using Modified Cincinnati scoring system.

Methods: Between November 2010 to May 2011, forty consecutive alternate patients, 20 in each group underwent arthroscopic assisted ACL reconstruction using BPTB graft or the QHT autograft. All the patients underwent standard ipsilateral arthroscopic ACL reconstruction procedure using the single incision AnteroMedial (AM) portal technique for BPTB graft or the QHT autograft done by a single surgeon. Patients were followed up regularly for a minimum period of 2 years.

Results: Mean age of the study participants was 28.43 years (SD: 5.48). Mean Modified Cincinnati score at the end of 2 years was $99.0 \%$ for QHT group and $98.8 \%$ for BPTB graft group $(p=0.48)$. The hamstring tendon group also had lower graft harvest site morbidity, as demonstrated by less kneeling pain at 2 years.

Conclusion: There was no significant functional difference as demonstrated by Modified Cincinnati score among patients undergoing QHT graft or the BPTB graft for ACL reconstruction at the end of 2 years.

Keywords: Anterior cruciate ligament, Arthroscopic ACL reconstruction, Bone-Patellar-Tendon-Bone graft, Modified Cincinnati scoring system, Quadruple hamstring tendon graft.
\end{abstract}

\section{Introduction}

An Anterior cruciate ligament (ACL) injured knee leads to change in lifestyle. ACL occupies a pivotal position in the knee; it along with other ligaments stabilizes the knee [1].Anterior cruciate ligament (ACL) tear is the most common ligamentous injury to the knee joint [1-5]. ACL injuries usually occur to the young, dynamic, bread winners of our society. It becomes a road block to their development, sometimes can lead to a career change in sports person, such is the impact of ACL injury.

The ACL is the primary stabilizer against anterior translation of the tibia on the femur and is important in counteracting rotation and valgus stress.ACL deficiency leads to knee instability[6]. This results in recurrent injuries and increased risk of intra- articular damage, especially the meniscus $[7,8]$. The goals of ACL reconstruction are to restore stability to the knee; allow the patient to return to normal activities, including sports; and to delay the onset of osteoarthritis with associated recurrent injuries to the articular cartilage and loss of meniscal functions [9-11]. During the past decade arthroscopically assisted techniques have been an accepted method of reconstructing the ACL [11-13].

It is said that there is little or no difference between BPTB graft and QHT grafts in terms of functional outcome after ACL reconstruction, despite greater laxity measurements in the hamstring tendon group patients [13]. This suggests that operating surgeon must decide how to select the appropriate graft for an individual patient. Graft choice, surgeon experience, correct graft position and post-operative rehabilitation confound the results of comparing various different methods of ACL reconstruction [14]. 
The objective of this study was to compare the functional outcome of surgeries performed to reconstruct the ACL using QHT graft and BPTB grafts using Modified Cincinnati scoring system[15,16].

\section{Materials and Methods}

This is a prospective randomized control study in which alternate consecutive patients underwent single incision AM portal arthroscopically assisted ACL reconstructions using either the BPTB graft or the QHT autograft between November 2010 and May 2011were followed up for a minimum period of 2 years.

Clinical diagnosis was made by performing Lachman test and Pivot shift test. MRI confirmed the diagnosis and also helped to identify any additional findings. The ideal candidate for surgery was a young healthy patient who experienced knee instability in daily activities, sports or wished to maintain his or her preinjury level of activities.

\subsection{Inclusion criteria}

All patients between the age of 18 to 45 years with symptomatic and clinical knee instability due to isolated ACL deficiency with or without meniscal tears.

\subsection{Exclusion criteria}

\subsubsection{Bilateral ACL deficiency}

2.2.2.Revision ACL surgery.

2.2.3. Previous knee operation.

2.2.4. Concomitant medical illness or geographic constraint that precluded follow-up evaluations.

All the surgeries were performed by the single surgeon to reduce the confounding factors and minimize bias which could hamper the functional outcome of the study.

All patients were operated under spinal anaesthesia in supine position, affected lower limb cleaned and draped. Tourniquet was used routinely. Once the ACL tear was confirmed after diagnostic arthroscopy, the graft was than harvested. Any meniscal tears requiring balancing was done during diagnostic arthroscopy. Patients undergoing meniscal repair were not considered for the study as the post-op protocol for such patients differed.

Both the gracilis and semitendinosus tendons were harvested using a $2.5-\mathrm{cm}$ incision made anteromedially on the proximal tibia starting approximately $4 \mathrm{~cm}$ distal to the joint line and $3 \mathrm{~cm}$ medial to the tibial tuberosity midway between the anterior and posterior cortex. A whip stitch was used to suture the tendons. Two different colour sutures were used for each of the tendons so as to identify each tendon separately and separate tension can be given at the time of graft fixation. We routinely use ethibond (dyed green) and vicryl (blue) for two tendons. They are then folded into four to make it quadruple graft. We do not suture the tendons together as we feel that keeping the tendons separately may mimic double bundles of the original ACL.

The BPTB graft was harvested from middle third of patellar tendon and contoured with the rongeurs so that it fits through the 10-mm trial sizer, ensuring that the complete graft would pass through the trial. The ends of the bone plug were rounded to make passage of the graft easier. The bone-tendon junction on the cancellous side of the graft at both ends was marked with a marker pen, and the total graft length was measured.

The ACL stump was visualized and partially debrided. The remnant stumps of the ACL are retained as much as possible so as to help in graft integration (biological fixation). We also believe that the torn stump may help in better proprioception of the knee as it has proprioceptors[17]. After the procedure, periosteal flap and the Sartorius fascia are sutured back over the tunnel to cover the screw head. The subcutaneous tissue was closed with interrupted 2-0 Vicryl sutures and the skin with staples.

Standard procedure for QHT and BPTB graft was followed with QHT graft fixed with $15 \mathrm{~mm}$ continuous loop endobutton (smith and nephew) on the femoral side and bio-absorbable screw (stryker) on the tibial side. Compression with an elastic crepe bandage was applied from toe till mid-thigh. The limb was immobilized in a knee brace.

For BPTB group the femoral tunnel was made through the antero-medial portal and graft was passed with the help of an artery forceps. Femoral screw was put from the antero-medial portal. We used bioabsorbable screws (stryker) for both femoral and tibial tunnels. For femoral side graft fixation the diameter of the screw used was equal to the diameter of the tunnel and for the tibial side we used a screw $1 \mathrm{~mm}$ more than the diameter of the tunnel.

All patients were followed up initially by operating surgeon. Patients were asked to follow the Simple and Effective Rehabilitation Protocol (SERP) developed by our institution (table 1)[18]. All final clinical testing and evaluations were performed by the other independent observer at the end of $3^{\text {rd }}, 6^{\text {th }}, 9^{\text {th }}, 12^{\text {th }}, 18^{\text {th }}$ month and at 24 months to eliminate potential bias. The assessment was done using the Modified Cincinnati Scoring System $[15,16]$. 


\section{Results}

Age of the patients in this study ranges from 20 years to 44 years. The mean age of the study participant was 28.43 (SD: 5.48) years. Majority of patients $(n=23)$ were between 20-29 years age group. Out of the 40 patients, 38 were male and 2 were female. Most common mechanism of injury was road traffic accident in 25 patients $(62.5 \%)$, followed by sports related injuries in 10 patients $(25 \%)$ and fall from height in 5 patients $(12.5 \%)$. Twenty four patients $(60 \%)$ sustained injury to right side as compared to 16 patients $(40 \%)$ who sustained injury to left knee. All the patients were followed up for a minimum period of 2 years. The mean modified Cincinnati knee score at the end of 2 years was $99.0 \%$ for QHT group and $98.8 \%$ for BPTB graft group $(\mathrm{p}=0.48)$. The hamstring tendon group also had lower graft harvest site morbidity, as demonstrated by less kneeling pain at 2 years.

Three patients had additional posterior horn medial meniscus tear which required balancing of the tear. Two patients in the hamstring group had serous discharge from the tibial graft harvest site for more than 3 weeks post-operative period. Culture from the discharge showed no growth. No knee effusion, local and systemic signs of infection was noted. Wound debridement under cover broad spectrum antibiotics was done for both cases. The wound subsequently healed in both the cases without affecting the rehabilitation protocol. One patient, in the QHT group had thigh muscle hypotrophy $(2.2 \mathrm{cms})$ compared to the opposite side which persisted even till 2 years of follow up which did not affect the functional outcome.

Table 1: Simple And Effective Rehabilitation Programme (SERP)

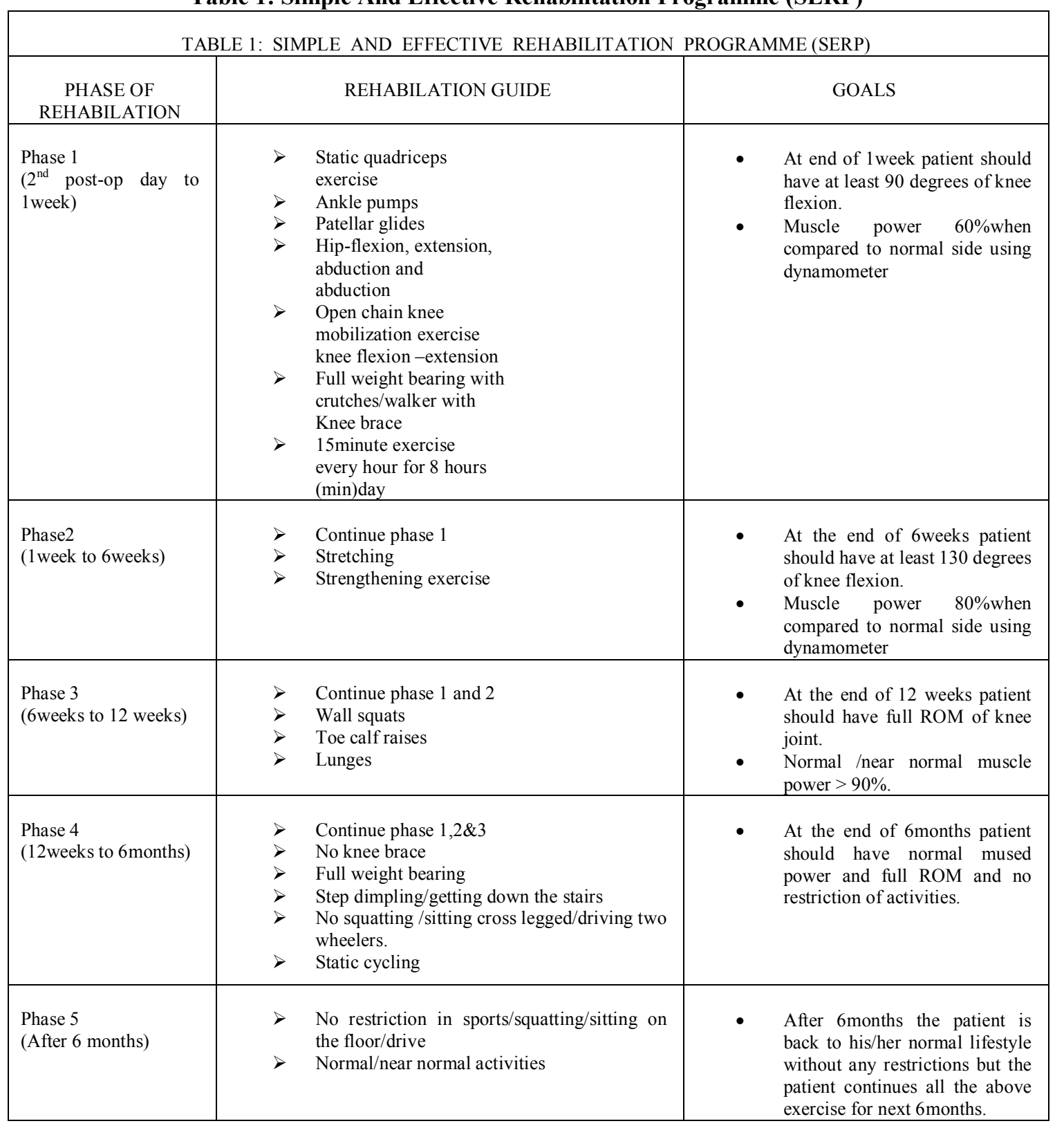


Table 2: Descriptive statistics showing the modified Cincinnati scores of BPTB graft and Hamstring graft procedures.

\begin{tabular}{|c|c|c|c|c|}
\hline $\begin{array}{l}\text { FOLLOW-UP } \\
\text { (MONTHS) }\end{array}$ & Graft & Mean & Std.Deviation(SD) & $\begin{array}{l}\text { Number of patients } \\
\text { (N) }\end{array}$ \\
\hline \multirow{3}{*}{ 6months } & ВРTB & 74.80 & 5.709 & 20 \\
\hline & STG & 76.80 & 3.205 & 20 \\
\hline & Total & 76.80 & 4.681 & 40 \\
\hline \multirow{3}{*}{ 9months } & ВРТВ & 88.30 & 4.646 & 20 \\
\hline & STG & 89.10 & 3.865 & 20 \\
\hline & Total & 88.70 & 4.238 & 40 \\
\hline \multirow{3}{*}{12 months } & BPTB & 98.25 & 1.789 & 20 \\
\hline & STG & 98.40 & 2.601 & 20 \\
\hline & Total & $\begin{array}{l}98.28 \\
\end{array}$ & 2.207 & 40 \\
\hline \multirow{3}{*}{18 months } & BPTB & 98.40 & 1.046 & 20 \\
\hline & STG & 98.60 & 1.603 & 20 \\
\hline & Total & 98.50 & 1.340 & 40 \\
\hline \multirow{3}{*}{24 months } & BPTB & 98.80 & 1.046 & 20 \\
\hline & STG & 99.00 & 0.875 & 20 \\
\hline & Total & 98.90 & 0.952 & 40 \\
\hline
\end{tabular}

\section{Discussion}

The BPTB and the QHT graft are the two most commonly used autografts for reconstruction [1923].The bone-patellar tendon-bone autograft has been widely accepted as the gold standard for ACL reconstruction with high success rate [24-26]. However, donor site morbidities and extensor mechanism problems associated with the use of bone-patellar tendon-bone have led to increasing popularity of the hamstring tendon graft which has advantages of low donor site morbidity, avoidance of extensor mechanism problems and better cosmoses.

There have been many prospective randomized control studies comparing two groups published in recent years. In a similar study, Corry, et al found that the two grafts did not differ in terms of clinical stability, range of motion and general symptoms. The hamstring tendon group also had lower graft harvest site morbidity.

In the study of arthroscopic anterior cruciate ligament reconstruction with BPTB graft, Akgun, et al [27] found that the best results could be obtained if the reconstruction was done in the sub-acute period between 3-5 weeks post-injury. The patients in the bone- patellar tendon bone group would have more desire to return to sports activity or higher functional demand than in the hamstring group, therefore higher expectation. Donor site morbidity is a major drawback of the BPTB graft. All patients in the BPTB group of the present study had experienced a disturbance of anterior knee sensation which continued for a period of time. In contrast, there was no sensory disturbance in the hamstring group. The hamstring group had also presumably better cosmoses. Results from these studies showed that the two groups had similar outcomes at the 2-5 year period.

In 2001, Yunes, et al [28] were the first to report a meta-analysis conducted from controlled trials of BPTB graft versus hamstring tendons for ACL reconstruction. They found that the patellar tendon graft patients had a greater chance of returning to pre-injury activity levels. They concluded that although both techniques yielded good results, patellar tendon reconstruction led to higher postoperative activity levels and greater static stability than hamstring reconstruction.

In 2003, using the same and extended numbers of controlled trial, Freedman, et al[29] found that the rate of graft failure in the patellar tendon group was significantly lower and a significant higher proportion of patients in the patellar tendon group had a difference of less than $3 \mathrm{~mm}$ on KT- 1000 arthrometer testing than in 
the hamstring tendon group. They concluded that patellar tendon autografts had a significantly lower rate of graft failure and resulted in better knee stability and increased patient satisfaction compared with hamstring tendon autografts. However, patellar tendon autograft reconstruction resulted in an increased rate of anterior knee pain.

Results of our study clearly showed that both BPTB graft and QHT grafts could effectively improve knee stability and functions after anterior cruciate ligament reconstruction. At follow-up evaluation, both groups had similar subjective outcome at the end 2 years. Long term results are awaited.

\section{Conclusion:}

The short term results of Arthroscopic ACL reconstruction using QHT graft and BPTB bone graft based on Modified Cincinnati scoring system were almost similar, with each having its own advantages and disadvantages. There is no significant difference between both the groups in terms of functional outcome.

\section{Acknowledgement}

We sincerely thank DR. RAVICHANDRAN, Lecturer, Department of biostatistics, Pondicherry Institute of Medical Sciences for having analysed the data statistically.

Consent: All patients have given their informed consent for the publication of this case series.

"The author(s) declare that they have no competing interests".

No funding received from any source for the study.

Ethical clearance from the ethical committee has been obtained for the above study.

\section{References:}

[1] Tanarat Boonriong MD, Niran Kietsiriroje MD, Arthroscopically Assisted Anterior Cruciate Ligament Reconstruction: Comparison of Bone-Patellar Tendon-Bone Versus Hamstring Tendon Autograft, J Med Assoc Thai Vol. 87 No.9 2004, 1100.

[2] Barry B Phillips. Campbell's operative orthopaedics. Tenth edition. chapter 48.

[3] Ian S. Corry, MD, FRCS(Orth), Jonathan M. Webb, FRCS(Orth), Amanda J. Clingeleffer, and Leo A. Pinczewski, FRACS, Arthroscopic Reconstruction of theAnterior Cruciate Ligament A Comparison of Patellar Tendon Autograft and Four-Strand Hamstring Tendon Autograft. Am. J. Sports Med. 1999; 27; 444

[4] Miyasaka KC, Daniel DM, Stone ML. the incidence of knee ligament injuries in the general population. Am J LneeSurg 1991;4:38 .

[5] Moyes FR, Bassett RW, Grood ES, Butler DL. Arthroscopy in acute traumatic hemarthrosis of the knee.Incidence of anterior cruciate tears and other injuries. J Bone Joint Surg (Am) 1980;62: 687-95,757.

[6] Levy IM, Torzilli PA, Warren RF. The effect of meniscectomy on anterior-posterior motion of the knee. J bone Joint $\operatorname{Surg}(\mathrm{Am}) 1982 ; 64: 883-8$.

[7] Jomha NM, Pinczewski LA, Clingeleffer A .Otto A. arthroscopic reconstruction of anterior cruciate ligament with patellar-tendon autograft and interference screw fixation. The results at seven years. J Bone Joint Surg (Br) 1999;81:775-9.

[8] Barrack RL, Bruckner JD, Knist J. Inman WS, Alexander AH. The outcome of non-operatively treated complete tears of the anterior cruciate ligament in active young adults. ClinOathop 1990; 259: 192-9.

[9] Dye SF, Wojtys EM, Fu FH, Fithian DC, Gilliquist J. Factors contributing to function of the knee joint after injury and reconstruction of the anterior cruciate ligament. In Zuckerman JD.Ed. Instructional Course Lecture. Rosemont , American Academy of Orthopaedic Surgery.1999;48: 185-98.

[10] Jorgensen U, Sonne-Holm U, Lauridsen F, Rosenklint A. Long term follow-up of meniscectomy in athletes. J Bone Joint Surg (Br) $1987 ; 69: 80-3$.

[11] Rangger C, KlestilT, Gloetzer W, Kemmler G, Benedetto KP. Osteoarthritis after arthroscopic partial meniscectomy. Am J Sports Med 1995;23:240-4

[12] MatjazSajovic , MD. VilibaldVengust MD, RadkoKomadina MD, RokTavcar, MD and KatjaSkaza, PT . A Prospective , Randomized Comparison of Semitendinosus and Gracilis Tendon Versus patellar Tendon Autografts for ACL Reconstruction five Year Follow-up. Am.J.Sports Med,2006;34;1933.

[13] Julian A. Feller,FRACS, and Kate E. Webster,PhD. A Randomized Comparison of patellar tendon and hamstring tendon ACL reconstruction. Am .j,Sports Med.2003;31;564

[14] Leo A, Pinczewski, FRACS, Jeffrey Lyman, MD, Lucy J.Salmon, PhD, VivianneJ.Russell, BSc (Biomed),Justin Roe ,FRACS, and James Linklater, FRANZCR. A 10 year Comoparison of Anterior Cruciate Ligamnet Reconstructions with hamstring Tendon and Patellar Tendon Autograft A Controlled, prospective trial. Am.J.Sports Med.2007;335;564.

[15] Noyes FR, Barber SD, Mooar LA. A rationale for assessing sports activity levels and limitations in knee disorders. Clin Orthop Relat Res. 1989 Sep;(246):238-49.

[16] Noyes FRed. The Cincinnati knee Rating System. Cincinnati, OH: Cincinnati Sports Medicine Research and Education Foundation; 1983.

[17] Mandeep S Dhillon, Kamal Bali, SharadPrabhakar. Proprioception in anterior cruciate ligament deficient knees and its relevance in anterior cruciate ligament reconstruction. Indian J Orthop. 2011 Jul-Aug; 45(4): 294-300.

[18] Shishir.S.M, Manoj Abraham M, Dr.Nikhil Joseph Martin, Dr.Kanagasabai.R, Dr.James J Gnanadoss. Simple and Effective Rehabilitation Programme (SERP) for patients undergoing Arthroscopic Anterior Cruciate Ligament (ACL) reconstruction in Indian scenario. Indian Journal of Physiotherapy and Occupational Therapy. 2013 October-December; Vol-7,number 4:222-228.

[19] Shaieb MD ,Kan DM, Chang SK, Marumoto JM and Richardson AB. A prospective randomized comparison of patellae versus semitendinosus and gracilis tendon autografts for anterior cruciate ligament reconstruction. Am J Sports Med 2002; 30; 214-20.

[20] Barrett GR, Noojin FK, Hartzog CW, Nash SR. Reconstruction of the anterior cruciate ligament in females. A comparison of hamstring versus patellar tendon autograft. Arthroscopy $2002 ; 18: 46-54$

[21] Ejerhed L, Kartus J. Sernet N, Kohler k, Karlsson J. Patellar tendon or semitendinous tendon autografts for anterior cruciate reconstruction : a prospective randomized study with a two year follow up. Am J sports Med 2003;31 : 19-25. 
[22] Jansson KA, Linko E, sandelin J, Harilainen A. A prospective randomized study of patellar versus hamstring tendon autografts for anterior cruciate ligament reconstruction. Am J sports Med 2003;31 : 12-8.

[23] Pinczewski LA, Deehan DJ, Salmon LJ. Russell VJ, and Clingeleffer A. A five year comparison of patellar tendon versus fourstrand hamstring tendon autograft for arthroscopic reconstruction of the anterior cruciate ligament. Am J sports Med 2002;30:52336

[24] BeynnonBD, Johnson RJ,Fleming BC, Kannus P, Kaplan M, samani J and Renstrom P. Anterior cruciate ligament replacement : comparison of bone-patellar tendon-bone grafts with two strand hamstring grafts. J Bone Surg (Am) 2002;84:1503-13.

[25] AgliettiP, BuzziR, Zaccherotti G, DeBise P, patellar tendon versus doubled semitendinosous and gracilis tendons for anterior cruciate ligament reconstruction. Am J Sports med 1994;22: 211-8.

[26] Marder RA, Raskind JR, Carroll M. Prospective evaluation of arthroscopically assisted for anterior cruciate ligament reconstruction. Patellar tendon versus semitendinosous and gracilis tendons. Am J Sports med 1991;19:478-84.

[27] Akgun I, et al. Central third bone-patellar tendon-bone arthroscopic anterior cruciate ligament reconstruction: a 4-year follow-up. J knee Surg. 2002 Fall;15(4):207-212.

[28] Yunes M, et al. Patellar versus hamstring tendons in anterior cruciate ligament reconstruction: A meta-analysis. Arthroscopy.2001 Mar;17(3):248-257.

[29] Freedman KB, et al. Arthroscopic anterior cruciate ligament reconstruction: a meta analysis comparing patellar tendon and hamstring tendon autografts. Am J Sports Med.2003 Jan-Feb;31(1):2-11.

\section{Figures:}

Figure 1: QHT graft after preparation. One end has been secured with $15 \mathrm{~mm}$ continuous loop Endobutton.

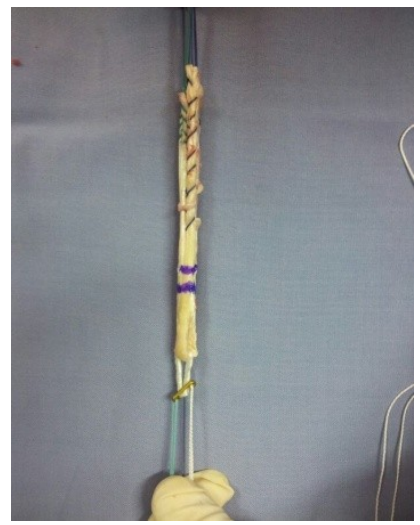

Figure 2: Skin closure after QHT graft procedure of right knee.

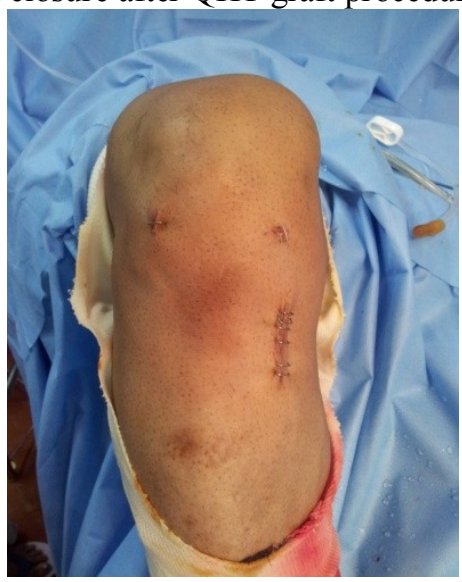


Figure 3 :Post Op X-ray lateral and antero-posterior view showing the endobutton on the lateral cortex of lateral femoral condyle.

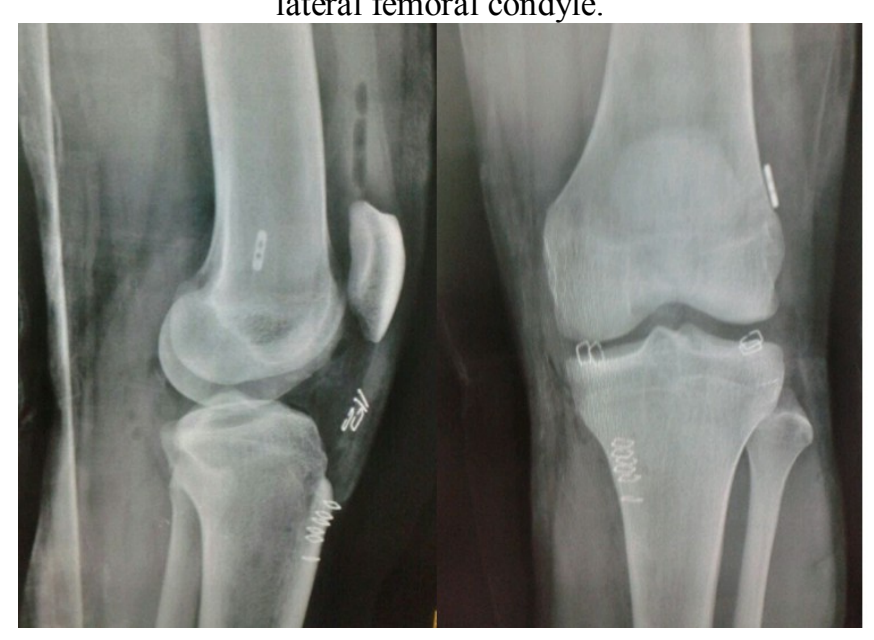

Figure 4: Harvested BPTB graft

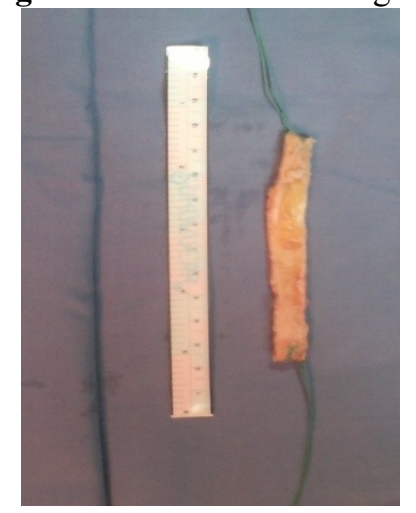

Figure 5: Skin closure after BPTB graft procedure of left knee.

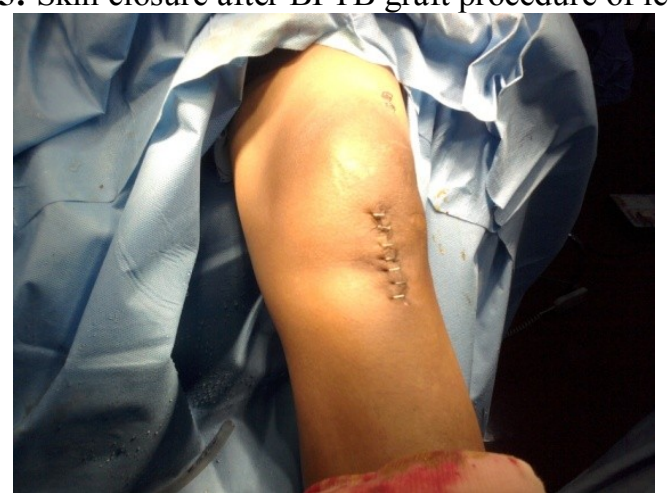


Figure 6: Post-op X-ray showing the tibial and femoral tunnels.

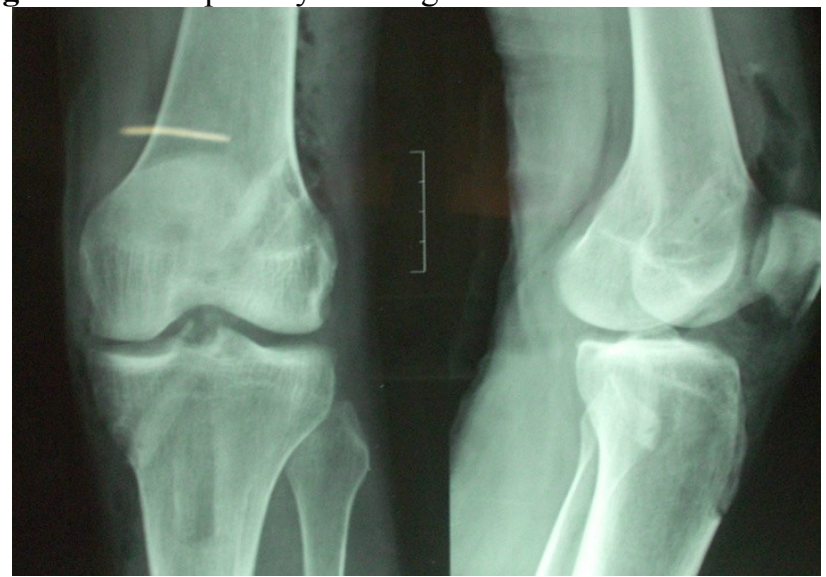

Figure 7: Arthroscopic view of the graft with knee in extension

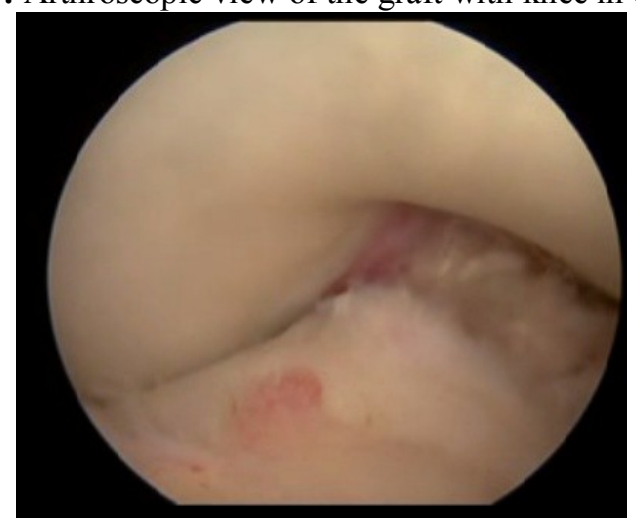

Figure 8: Arthroscopic view of the graft with knee in flexion

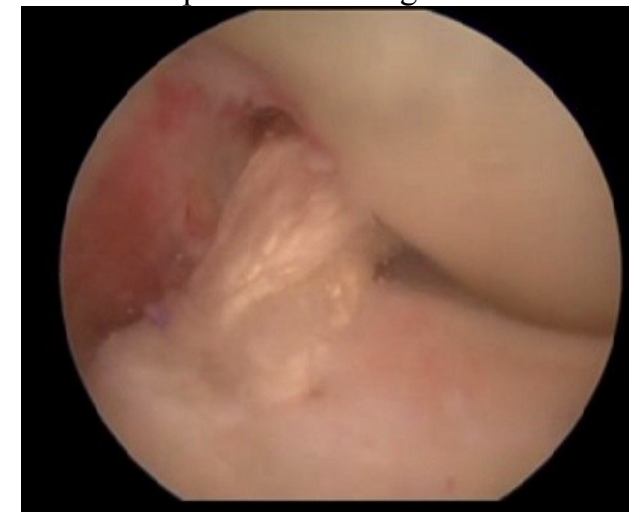

